\title{
LAMINAR FLOW BEHIND A TWO-DIMENSIONAL GRID
}

\author{
BY L. I. G. KOVASZNAY
}

Communicated by Sir Geoffrey TaYlor

Received 20. May 1947

\section{INTRODUOTION}

The production of a 'wake' behind solid bodies has been treated by different authors. S. Goldstein (1) and S. H. Hollingdale (2) have discussed the laminar wake behind a flat plate, while the wake of a two-dimensional grid has been treated for the turbulent case alone using L. Prandtl's 'Mischungsweg'. theory by E. Anderlik and Gran Olsson (3), (4).

This paper presents an exact two-dimensional solution of the Navier-Stokes equations with a periodicity in one direction, which may represent the wake of a twodimensional grid.

SOLUTION OF THE EQUATIONS OF MOTION

The equations of a two-dimensional motion are

$$
\begin{aligned}
& \frac{\partial u^{\prime}}{\partial t^{\prime}}+\left(u_{0}+u^{\prime}\right) \frac{\partial u^{\prime}}{\partial x^{\prime}}+v^{\prime} \frac{\partial u^{\prime}}{\partial y^{\prime}}=-\frac{1}{\rho^{\prime}} \frac{\partial p^{\prime}}{\partial x^{\prime}}+\nu \nabla^{\prime 2} u^{\prime}, \\
& \frac{\partial v^{\prime}}{\partial t^{\prime}}+\left(u_{0}+u^{\prime}\right) \frac{\partial v^{\prime}}{\partial x^{\prime}}+v^{\prime} \frac{\partial v^{\prime}}{\partial y^{\prime}}=-\frac{1}{\rho^{\prime}} \frac{\partial p^{\prime}}{\partial y^{\prime}}+\nu \nabla^{\prime 2} v^{\prime},
\end{aligned}
$$

where $u_{0}$ is the average velocity in the $x$ direction,

$u_{0}+u^{\prime}\left(x^{\prime}, y^{\prime}\right)$ is the local velocity in the $x$ direction,

$v^{\prime}\left(x^{\prime}, y^{\prime}\right)$ is the local velocity in the $y$ direction,

$p^{\prime}\left(x^{\prime}, y^{\prime}\right)$ is the pressure,

$\nu$ is the kinematic viscosity, and

$$
\nabla^{\prime 2}=\frac{\partial^{2}}{\partial x^{\prime 2}}+\frac{\partial^{2}}{\partial y^{\prime 2}} .
$$

Eliminating the pressure and introducing the vorticity

$$
\omega^{\prime}=\frac{\partial v^{\prime}}{\partial x^{\prime}}-\frac{\partial u^{\prime}}{\partial y^{\prime}},
$$

we obtain from equations $(1 a)$ and $(1 b)$

$$
\frac{\partial \omega^{\prime}}{\partial t^{\prime}}+\left(u_{0}+u^{\prime}\right) \frac{\partial \omega^{\prime}}{\partial x^{\prime}}+v^{\prime} \frac{\partial \omega^{\prime}}{\partial y^{\prime}}=\nu \nabla^{\prime 2} \omega^{\prime} .
$$

Introduce dimensionless quantities using $u_{0}$ as reference velocity and $M$ (the spacing of the grid) as reference length; thus the Reynolds number of the grid is $R=M u_{0} / \nu$ and

$$
\begin{aligned}
& x=\frac{x^{\prime}}{\bar{M}}, \quad y=\frac{y^{\prime}}{M}, \quad t=\frac{t^{\prime} u_{0}}{M}, \\
& u=\frac{u^{\prime}}{u_{0}}, \quad v=\frac{v^{\prime}}{u_{0}}, \quad \omega=\frac{\omega^{\prime} M}{u_{0}} .
\end{aligned}
$$


So equation (2) becomes

$$
\frac{\partial \omega}{\partial t}+(1+u) \frac{\partial \omega}{\partial x}+v \frac{\partial \omega}{\partial y}=\frac{1}{R}\left(\frac{\partial^{2} \omega}{\partial x^{2}}+\frac{\partial^{2} \omega}{\partial y^{2}}\right)
$$

for steady motion $\quad \frac{\partial^{2} \omega}{\partial x^{2}}-R \frac{\partial \omega}{\partial x}+\frac{\partial^{2} \omega}{\partial y^{2}}-R\left(u \frac{\partial \omega}{\partial x}+v \frac{\partial \omega}{\partial y}\right)=0$.

The continuity equation

$$
\begin{aligned}
& \frac{\partial u}{\partial x}+\frac{\partial v}{\partial y}=0, \\
& \omega=\frac{\partial v}{\partial x}-\frac{\partial u}{\partial y},
\end{aligned}
$$

and the definition of

represent with equation (3) the whole set of equations to be solved.

If the change of velocity were very small compared with the average velocity, we could neglect the two quadratic terms. To avoid this we try to find a solution for which these two quadratic terms vanish. Another type of flow for which the 'inertia terms' are cancelled is given by G. I. Taylor (5).

Let us introduce a stream function $\psi$, such that

$$
\begin{aligned}
u & =\frac{\partial \psi}{\partial y}, \\
v & =-\frac{\partial \psi}{\partial x}, \\
\omega & =-\nabla^{2} \psi .
\end{aligned}
$$

Let the stream function be of the simplest form

$$
\psi=f(x) \sin 2 \pi y
$$

The quadratic terms of equation (3) vanish when

Substituting (5) into (6), we have

$$
u \frac{\partial \omega}{\partial x}+v \frac{\partial \omega}{\partial y}=0
$$

$$
f^{\prime} f^{\prime \prime}-f f^{\prime \prime \prime}=0
$$

and if any of the derivatives are not identically zero,

Integrating $(6 b)$, we obtain

$$
\frac{f^{\prime \prime \prime}}{f^{\prime \prime}}=\frac{f^{\prime}}{f}
$$

where $k$ is an arbitrary constant (real or complex).

Integrating (6c), we have

$$
\begin{gathered}
f(x)=A e^{k x} \\
\psi=A e^{k x} \sin 2 \pi y .
\end{gathered}
$$

Hence the quadratic terms vanish if $f$ is chosen to be an exponential function of $x$. The sum of such functions does not fulfil equation (6).

When the condition (6) is fulfilled equation (3) becomes linear,

and substituting

$$
\frac{\partial^{2} \omega}{\partial x^{2}}-R \frac{\partial \omega}{\partial x}+\frac{\partial^{2} \omega}{\partial y^{2}}=0,
$$

$$
\omega=g(x) \sin 2 \pi y,
$$


60

we obtain

Solving (9), we have

where

L. I. G. KovasznaY

$$
\begin{gathered}
g^{\prime \prime}-R g^{\prime}-4 \pi^{2} g=0 . \\
g=c_{1} e^{\lambda_{1} x}+c_{2} e^{\lambda_{2} x}, \\
\lambda_{1,2}=\frac{R}{2} \pm \sqrt{ }\left(\frac{R^{2}}{4}+4 \pi^{2}\right) .
\end{gathered}
$$

The vorticity from $(8)$ and $(9 a)$ is thus

also from $(6 e)$

$$
\begin{aligned}
& \omega=\left(c_{1} e^{\lambda_{1} x}+c_{2} e^{\lambda_{2} x}\right) \sin 2 \pi y ; \\
& \omega=A\left(4 \pi^{2}-k^{2}\right) e^{k x} \sin 2 \pi y .
\end{aligned}
$$

Comparing $(9 c)$ with $(9 d)$ we see that two solutions are possible, namely

(i) $k=\lambda_{1}, \quad c_{1}=-R \lambda_{1} A, \quad c_{2}=0$,

(ii) $k=\lambda_{2}, \quad c_{1}=0, \quad c_{2}=-R \lambda_{2} A$.

If the Reynolds number is high enough, we may approximate to the root terms in the expression for $\lambda_{1}$ and $\lambda_{2}(9 b)$. Then simply

$$
\begin{gathered}
\lambda_{1}=R, \\
\lambda_{2}=-\frac{4 \pi^{2}}{R} .
\end{gathered}
$$

Fig. 1 shows the exact variation of $\lambda_{1}$ and $\lambda_{2}$ with the Reynolds number. The asymptotic values for $\lambda_{1}$ and $\lambda_{2}$ are shown with dotted lines in the diagram. When $R>30$, the simpler formulae may be used. If the Reynolds number is very small $(R<1)$ the absolute values of $\lambda_{1}$ and $\lambda_{2}$ tend to $2 \pi$.

The stream function of the average flow is $\psi_{0}=y$, so the total stream function is

$$
\psi_{0}+\psi=y+A e^{\lambda x} \sin 2 \pi y .
$$

The constant $A$ may be determined by fixing the stagnation point of the flow. If we choose the stagnation point $\left(u+u_{0}=0, v=0\right)$ at $x=0$, we get $A=-1 / 2 \pi$.

Hence we have all the functions needed to described the flow:

$$
\begin{gathered}
\psi+\psi_{0}=y-\frac{1}{2 \pi} e^{\lambda x} \sin 2 \pi y, \\
1+u=1-e^{\lambda x} \cos 2 \pi y, \\
v=-\frac{\lambda}{2 \pi} e^{\lambda x} \sin 2 \pi y, \\
\omega=\lambda R e^{\lambda x} \sin 2 \pi y .
\end{gathered}
$$

The streamlines of the two types of flow are shown in Figs. 2 and 3. Fig. 2 corresponds to $\lambda_{2}$ and represents a flow past the grid $(R=40)$. A pair of bound eddies occur behind the single elements of the grid. The streamlines become parallel and equidistant at infinity downstream as shown by the short lines on the right side of the figure. The dotted streamlines correspond to the half-value of stream function between two full lines.

When the Reynolds number increases, the whole flow pattern is extended uniformly in the direction of main flow.

Fig. 3 shows the other solution using $\lambda_{1}(R=40)$. The rate of change of the flow is here very great. The picture may represent a flow of alternating vortices superposed 

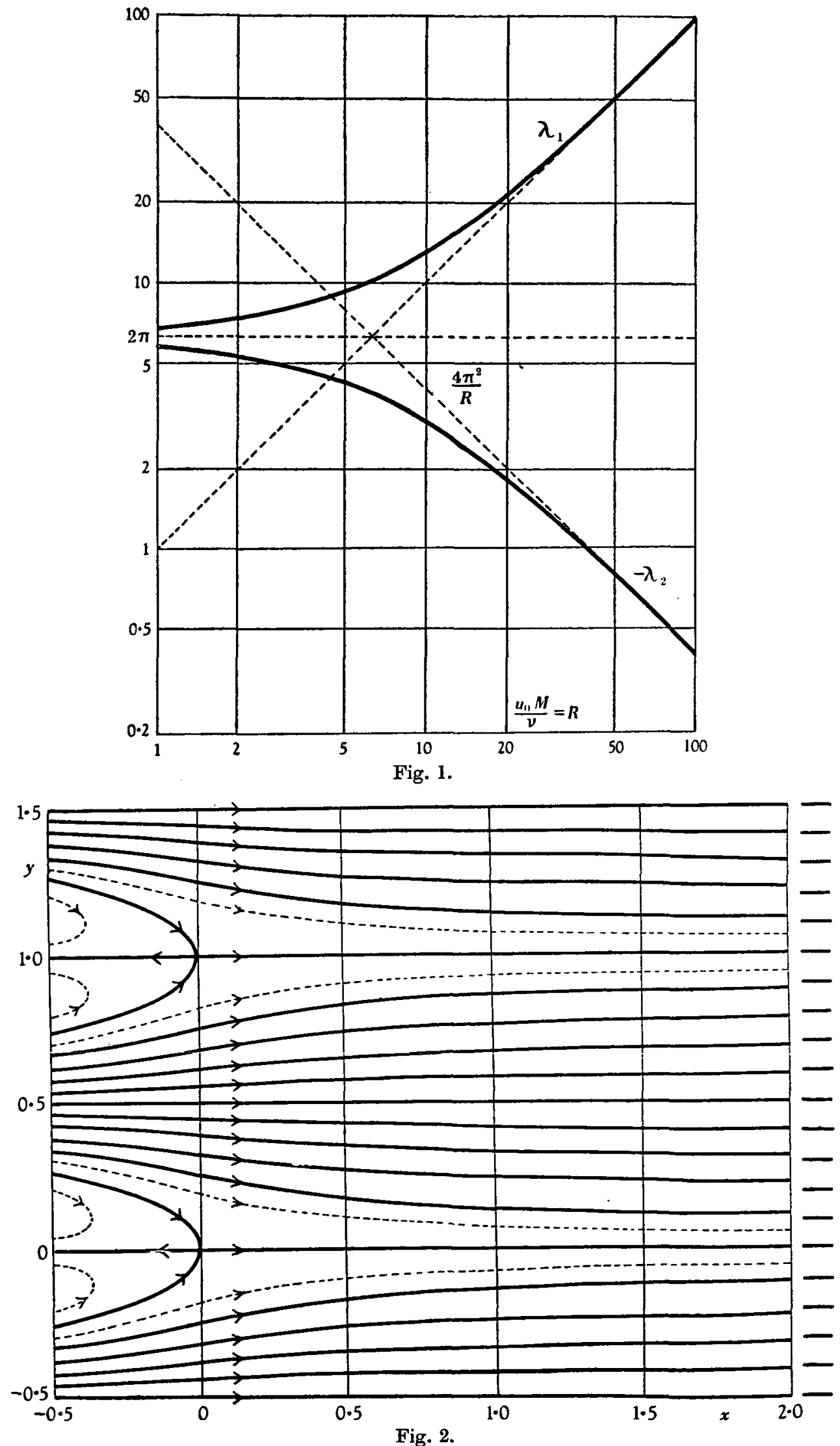
on a main flow perpendicular to their plane. With increasing Reynolds number the scale in the direction of main flow contracts.

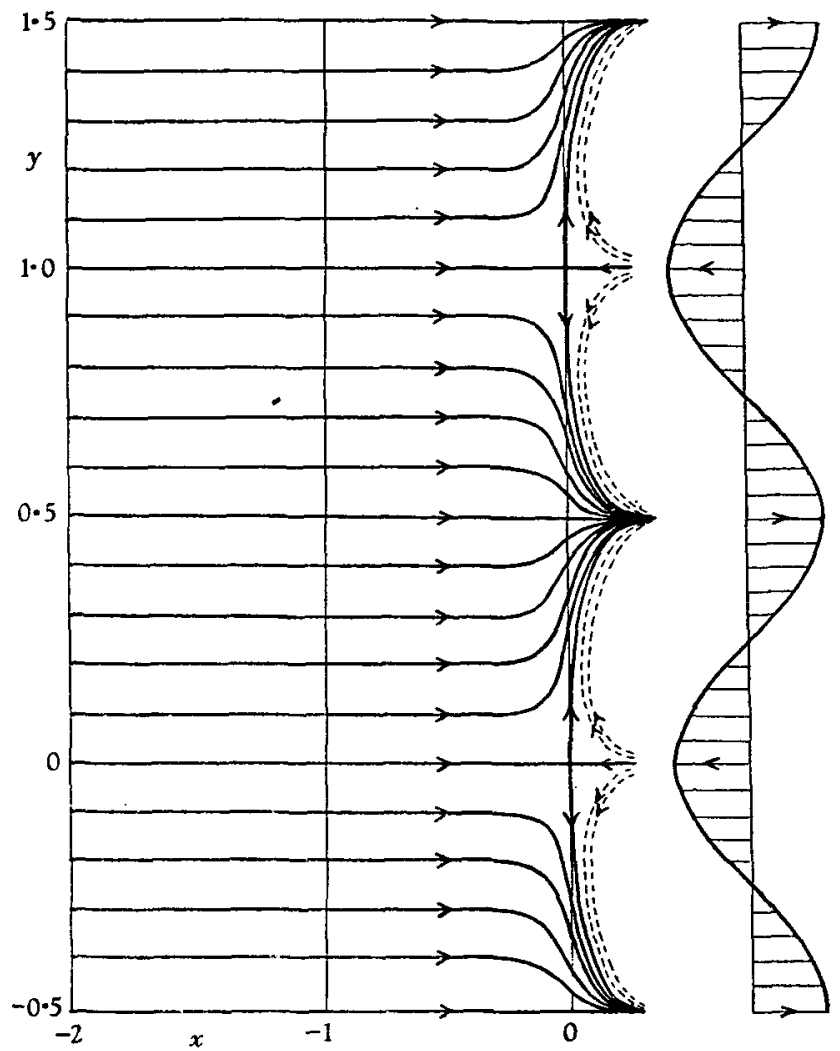

Fig. 3.

Conclusion

A simple exact solution of the Navier-Stokes equations, which may describe the motion behind a grid, consisting of equally spaced parallel rods or strips, has been found.

The solution does not give information in the plane of the grid, but seems to describe the flow quite closely behind it. The solution gives a representation of the pairs of bound eddies and gives the law of decay for the individual wakes of the elements of the grid for any Reynolds number.

The problem was suggested by Prof. Sir Geoffrey Taylor to whom I am greatly indebted for his advice and criticism. Thanks are due to the British Council for granting me a research scholarship.

\section{REFERENCES}

(1) Goldstein, S. Proc. Roy. Soc. A, 142 (1933), 563-73.

(2) Hollingdale, S. H. Phil. Mag. 29 (1940), 209-57.

(3) Arderitk, E. Mag. Tud. Akad. Mat. Term. Tud. Ertesitöje, 52 (1936), 54.

(4) Orsson, Gran Z. angew. Math. Mech. 16 (1936), 257-74.

(5) TAYLOR, G. I. Phil. Mag. 46 (1923), 671-4.

JoHNs Hopkins UnTVERSITY,

BaLTiMORE, U.S.A. 